\section{Identification and evaluation of antimicrobial resistance of enterococci isolated from raw ewes' and cows' milk collected in western Sicily: a preliminary investigation}

\author{
Marisa Palmeri, ${ }^{1}$ Isabella Mancuso, ${ }^{1}$ \\ Raimondo Gaglio, ${ }^{3}$ Luigi Arcuri, ${ }^{2}$ \\ Santino Barreca, ${ }^{1}$ Pietro Barbaccia, ${ }^{3}$ \\ Maria Luisa Scatassa ${ }^{1}$ \\ ${ }^{1}$ Institute for Experimental Veterinary \\ Medicine of Sicily A. Mirri, Palermo; \\ ${ }^{2}$ Local Health Unit, Palermo; \\ ${ }^{3}$ Department of Agricultural, Food and \\ Forest Sciences, University of Palermo, \\ Palermo, Italy
}

\begin{abstract}
The present work was carried out to investigate the Antimicrobial Resistance (AMR) of enterococci isolated from raw ewes' and cows' milk. The samples were collected from eighteen semi-extensive dairy sheep and cow farms throughout western Sicily. Plate counts, carried out on Rapid Enterococcus Agar commonly used to detect food enterococci, revealed a maximal enterococcal concentration of approximately 4.58 Log Colony Forming Unit $(\mathrm{CFU}) / \mathrm{mL}$. Colonies were isolated and differentiated based on genetic analysis by Randomly Amplified Polymorphic DNA (RAPD)-PCR. Thirty-eight different strains were identified. Analysis by a species-specific multiplex PCR assay grouped the strains into three Enterococcus species such as Enterococcus durans, Enterococcus faecalis and Enterococcus faecium. The 38 strains were also investigated for their antimicrobial resistance by a phenotypic approach. All 38 Enterococcus displayed resistance to at least one or more of the antimicrobials tested confirmed that the dairy enterococci could be a vector for the dissemination of antimicrobial resistance. This work showed that enterococci with AMR traits are commonly present in semiextensive dairy sheep and cow farms of western Sicily pointed out the relevance of informing dairy makers and veterinary regarding the antimicrobial use in order to mitigate problems of public health and veterinary medicine.
\end{abstract}

\section{Introduction}

Enterococci are a group of Lactic Acid Bacteria (LAB) that includes pathogenic, spoilage, and pro-technological microorganisms. They are widely distributed in nature, as they are present in several foods, in particular in those of animal origin (Franz, Holzapfel, and Stiles 1999; Gaglio et al., 2016a). These bacteria are also an integral component of humans and animals' gastrointestinal microflora (Mannu et al., 2003) and their presence in food, especially in dairy production, is a consequence of fecal contamination (Franciosi, Settanni, Cologna, Cavazza, and Poznanski, 2011).

Enterococci represent a part of the common LAB community present in milk and they were found in wooden equipment, animal rennet and in different typology of traditional cheeses (Cruciata, Gaglio, Todaro, and Settanni, 2019). Enterococci play an important role during the production of cheese, contributing to the development of the aromatic and organoleptic characteristics due to their proteolytic and lipolytic activities (Giraffa, 2002), and to extend their shelf life (Foulquiè Moreno, Sarantinopoulos, Tsakalidou, and De Vuyst, 2006). Different enterococci have been reported to produce bacteriocin-like inhibitory substance able to inhibit, in vitro and in vivo, pathogenic bacteria such as Listeria monocytogenes (Macaluso, Fiorenza, Gaglio, Mancuso, and Scatassa, 2016; Scatassa et al., 2017), to degrade biogenic amines (Guarcello et al., 2016a) and to protect against infections by promoting the maturation of the host's immune system (Fernández et al., 2012). To this purpose, some of them are being used as components of cheese adjunct cultures (Guarcello et al., 2016b). On the other hand, in the last decades enterococci, unlike other group of LAB, are not more considered Generally Recognized As Safe (GRAS), an essential condition for food production and moreover they are not included in the Quality Presumption of Safety (QPS) list of the European Food Safety Authority (EFSA BIOHAZ Panel, 2016). However, up until today, there are not evidence about the transmission of enterococci infection due to the consumption of food containing enterococci (Gaglio et al., 2016b). The ability of these bacteria to determine human infections is mainly imputable to virulence and Antimicrobial Resistance (AMR) traits. Regarding AMR, enterococci are intrinsically resistant to many antimicrobial agents and show the ability to transfer AMR to other microorganisms through mechanisms of plasmid conjugation that might lead to the generation of multiple antibiotic resis-
Correspondence: Maria Luisa Scatassa, Istituto Zooprofilattico Sperimentale della Sicilia A. Mirri, Palermo, Italy.

Tel.: +39 091 6565300; Fax +390916565332.

Email: luisa.scatassa@izssicilia.it

Key words: Enterococcus; antimicrobial resistance; sheep milk; cow milk.

Acknowledgments: The Authors thank B. Ducato for her precious collaboration.

Conflict of interests: The authors declare no conflict of interest.

Funding: This work was financed by the Italian Ministry of Health Research Project RC 06/16 Valutazione del rischio associato alla presenza di enterococchi antibiotico resistenti nei prodotti lattiero-caseari e sviluppo di metodologie che consentano la preservazione delle produzioni (CUP H76JI7000480001).

Received for publication: 16 July 2019.

Revision received: 20 January 2020.

Accepted for publication: 4 February 2020.

This work is licensed under a Creative Commons Attribution-NonCommercial 4.0 International License (CC BY-NC 4.0).

(C) Copyright: the Author(s), 2020

Licensee PAGEPress, Italy

Italian Journal of Food Safety 2020; 9:8406

doi:10.4081/ijfs.2020.8406

tance genes (Giraffa, 2002). As a matter of fact, the enterococci present in dairy products can be a possible intermediate vehicle for the transmission of antimicrobial resistance both to other commensal strains of the human gastrointestinal tract belonging to the same species than to different genera and pathogenic microorganisms (Guzman Prieto et al., 2016). Normally, in Mediterranean regions the extensive and semi-extensive breeding systems the animals are mainly raised on native pastures, forage crops or stubbles, and receive as supplements commercial concentrates, grains, hay or silages in periods of limited availability of green grass (Sitzia et al., 2015). This type of management, if well applied, allows to reduce stress factors and the presence of diseases with consequent reduced use of antimicrobial agents.

With this in mind, the present work was carried out in order to evaluate the antimicrobial resistance of enterococci isolated from different samples of ewes' and cows' milk collected from different Sicilian semiextensive dairy sheep and cow farms. Enterococci population in the milk samples were enumerated, isolated, identified and evaluated for antimicrobial resistance traits. 


\section{Materials and Methods}

\section{Sample collection}

Eighteen bulk milk samples of cow $(n=3)$ ewe $(n=15)$ were collected from semi-extensive dairy sheep and cow farms throughout western Sicily (Agrigento, Palermo and Trapani provinces) from December 2018 to May to 2019 (Table 1). The eighteen farms chosen randomly were characterized by medium herds of 200-250 milking ewe's and 20-30 milking cows. The sheep reared were of the Valle del Belice breed, while the cows belonged of the Simmental, Brown and Frisian breeds. Just after sampling, all samples were placed into a portable fridge and transferred to the Laboratory of Centro Latte e Lotta alle Mastiti (Istituto Zooprofilattico Sperimentale della Sicilia Adelmo Mirri, Palermo, Italy) where they were immediately subjected to microbiological investigations. The bulk milk samples from each farm were collected in duplicate at twoweek intervals.

\section{Microbiological analysis and isolation}

All 18 samples were serially diluted in physiological solution $(0.9 \% \mathrm{w} / \mathrm{v} \mathrm{NaCl})$. Cells suspensions were than inoculated on Rapid Enterococcus Agar (REA) purchased from Bio-Rad (Hercules, CA, USA) and aerobically incubated for $48 \mathrm{~h}$ at $37^{\circ} \mathrm{C}$. Plate counts were carried out in duplicate.

Gram-positive and catalase-negative bacterial cultures, presumptively considered enterococci, were obtained by randomly picking between five and ten colonies from the highest dilution plates. The isolates were purified by successive sub-culturing, and stored in Medium 17 (M17) broth media (Oxoid, Milan, Italy) containing $20 \%$ glycerol $(\mathrm{v} / \mathrm{v})$ at $-80^{\circ} \mathrm{C}$ until further analysis.

\section{Genotypic differentiation and identi- fication}

Genomic DNAs of presumptive enterococci isolates cultures were extracted using the InstaGene Matrix kit (Bio-Rad) following the manufacturer's instructions. Cells were harvested after overnight growth in MRS (Man Rogosa e Sharpe) broths at $37^{\circ} \mathrm{C}$ and washed in distilled $\mathrm{H}_{2} \mathrm{O}$. The crude cell extracts were used as templates for PCRs.

Strains were differentiated by Random Amplification of Polymorphic DNA (RAPD)-PCR analysis in a $30 \mu$ reaction volume with the primers M13, AB111 and AB106 used singly as reported by Gaglio et al. (2017).

The RAPD patterns were analysed using Gelcompare II software version 6.5 (Applied-Maths, Sint, Marten-Latem, Belgium).

The unequivocal identification of the strains belonging to the Enterococcus genus was obtained by applying the multiplex PCR assay on the sodA gene as described by Jackson, Fedorka-Cray, and Barrett (2004) with primers DU1, DU2, FL1, FL2, FM1 and FM2 specific for Enterococcus durans, Enterococcus faecalis and Enterococcus faecium, CA1, CA2, MU1, MU2, HI1 and HI2 specific for Enterococcus casseliflavus, Enterococcus mundtii and Enterococcus hirae.

\section{Antimicrobial susceptibility test}

The different enterococci strains were characterized for their susceptibility to different antimicrobial compounds commonly used for the treatment of human and animal infections (Gaglio et al., 2016b) by the disk diffusion method according to the Clinical and Laboratory Standards Institute guidelines (CLSI, 2017). The inocula were prepared by micro-dilution method suspending colonies in $5 \mathrm{ml}$ of physiological solution $(0.9 \% \mathrm{NaCl}, \mathrm{w} / \mathrm{v})$ until reaching a standard density of $0.5 \mathrm{McF}$ arland. The cell suspensions were swabbed for confluent growth onto Mueller Hinton agar with defibrinated $5 \%$ sheep blood and $6 \mathrm{~mm}$ filter paper discs were placed on the surface of the test medium and incubated aerobically at $37^{\circ} \mathrm{C}$ for 18-24h.

Fourteen antimicrobial compounds belonged to 11 families: penicillins [peni- cillin G (P- $10 \mathrm{UI}$ ) and ampicillin (AMP-10 $\mu \mathrm{g})$ ]; glycopeptides [vancomycin (VA-30 $\mu \mathrm{g})$ ]; macrolides [erythromycin $(\mathrm{E}-15 \mu \mathrm{g})$ ]; tetracyclines [tetracycline (TE-30 $\mu \mathrm{g})$ ]; fluoroquinolone [ciprofloxacin (CIP-5 $\mu \mathrm{g})$ and levofloxacin $(\mathrm{LEV}-5 \mu \mathrm{g})$ ]; phenicols [chloramphenicol $(\mathrm{C}-30 \mu \mathrm{g})]$; streptogramins [quinupristin/dalfopristin (QD-15 $\mu \mathrm{g})$ ]; oxazolidinones [linezolid (LZD-30 $\mu \mathrm{g})]$; aminoglycosides [high level gentamicin $(\mathrm{CN}-120 \mu \mathrm{g})$ and streptomycin $(\mathrm{S}-10 \mu \mathrm{g})]$; sulphonamides [sulphamethoxazole/ trimethoprim (SXT- $25 \mu \mathrm{g})]$; riphampicine [rifampicin (RD- $30 \mu \mathrm{g})$ ] were tested.

After incubation, each strain was classified as Susceptible (S), Intermediate (I) or Resistant (R) based on the inhibition zone diameters according to the CLSI guidelines (CLSI, 2017). All antibiotics were purchased from Oxoid.

\section{Statistical analyses}

Statistical analyses of microbiological counts performed between the eighteen bulk milk samples were conducted using STATISTICA software (StatSoft Inc., Tulsa, OK, USA). Microbial data were converted to the $\log$ scale before statistical elaborations. The post-hoc Tukey's multiple range whereas Student's/-test was applied to determine the significance and the difference between means values of eighteen bulk milk samples. A p $<0.05$ was deemed significant.

Table 1. Milk samples.

\begin{tabular}{llcc} 
Sample & City of dairy factory & Province & Type of Milk \\
M1 & Castronovo di Sicilia & Palermo & Bovine \\
M2 & Castronovo di Sicilia & Palermo & Bovine \\
\hline M3 & Cammarata & Agrigento & Bovine \\
M4 & Collesano & Palermo & Ovine \\
\hline M5 & Gangi & Palermo & Ovine \\
M6 & Partinico & Palermo & Ovine \\
\hline M7 & Alimena & Palermo & Ovine \\
M8 & Gangi & Palermo & Ovine \\
\hline M9 & Gibellina & Trapani & Ovine \\
M10 & Menfi & Agrigento & Ovine \\
\hline M11 & Contessa Entellina & Palermo & Ovine \\
M12 & Partanna & Trapani & Ovine \\
\hline M13 & Santa Margherita di Belice & Agrigento & Ovine \\
M14 & Santa Margherita di Belice & Agrigento & Ovine \\
\hline M15 & Salemi & Trapani & Ovine \\
M16 & Gangi & Palermo & Ovine \\
\hline M17 & Gangi & Palermo & Ovine \\
M18 & Alimena & Palermo & Ovine \\
\hline
\end{tabular}




\section{Results and Discussion}

Microbiological analyses, differentiation and identification of enterococci

Figure 1 shows the viable counts of the enterococci harbored on milk samples. The levels of these microrganisms were in the range of 2.40-4.58 showing statistical significant difference $(p<0.05)$ between the samples object of investigation. With the exception of samples M3, M4, M5 and M14 the levels of enterococci detected on REA were at approximately 3-4 Log CFU/mL. Similar levels have been reported for raw ewes' milk used for traditional cheese productions (Gaglio et al., 2019a).

A total of 72 colonies were collected from 18 bulk milk samples. All cultures were inspected microscopically and after Gram and catalase test, 66 coccus-shaped Gram-positive and catalase negative cultures were subjected to RAPD analysis in order to perform the strain typing. As reported in the dendrogram (Figure 2), the combination of the three RAPD patterns of each isolate indicated that the enterococcal community isolated from raw ewes' and cows' milk was composed of 38 different strains. These strains were further analysed by a species-specific multiplex PCR assay, which identified $17 \mathrm{E}$. faecalis, $12 \mathrm{E}$. faeci$u m$ and $9 E$. durans. The dendrogram clearly showed that, except for the stain 3246 ( $E$. durans) and 3261 (E.faecium), all the other strains grouped per species.

All Enterococcus species identified are commonly associated with raw milk and cheeses (Franciosi et al., 2011; Gaglio et al., 2019b), including stretched cheeses (Gaglio et al., 2019a) and wooden vats used for cheese making in Italy (Cruciata et al., 2018) and France (Licitra et al., 2007).

\section{Antibiotic susceptibility test}

The 38 Enterococcus strains were tested for their antimicrobial susceptibility to 14 antimicrobial compounds by the disk diffusion method according to the Clinical and Laboratory Standard Institute guidelines (CLSI, 2017). Patterns of antibiotic resistance with regards to species and source of isolation of the 38 strains are reported in Table 2. The results of antibiotic susceptibility test relatively to ampicillin and gentamicin are not reported in table, because no strains was scored resistant. All 38 Enterococcus displayed resistance to at least one or more of the antimicrobials tested. In particular, between the 38 strains, five $(13.2 \%)$ showed multidrug-resistant phenotype (resistance to at least three antibiotics).

None of the Enterococcus species iden-

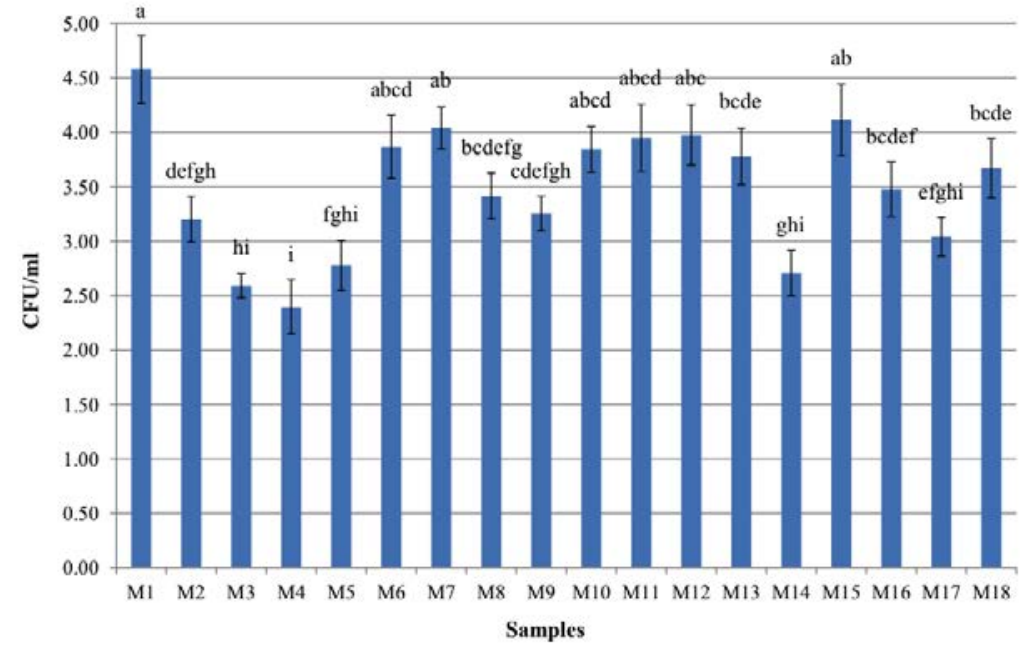

Results indicate mean values \pm standard deviation of four determinations (carried out in duplicate for two independent sampling). Different superscript letters indicate significant differences between mean values of microbial concentrations according to Tukey's test among the samples for $\mathrm{p}<0.05$

Figure 1. Microbiological concentrations of Enterococcus spp. (CFU/mL) in raw milk samples.

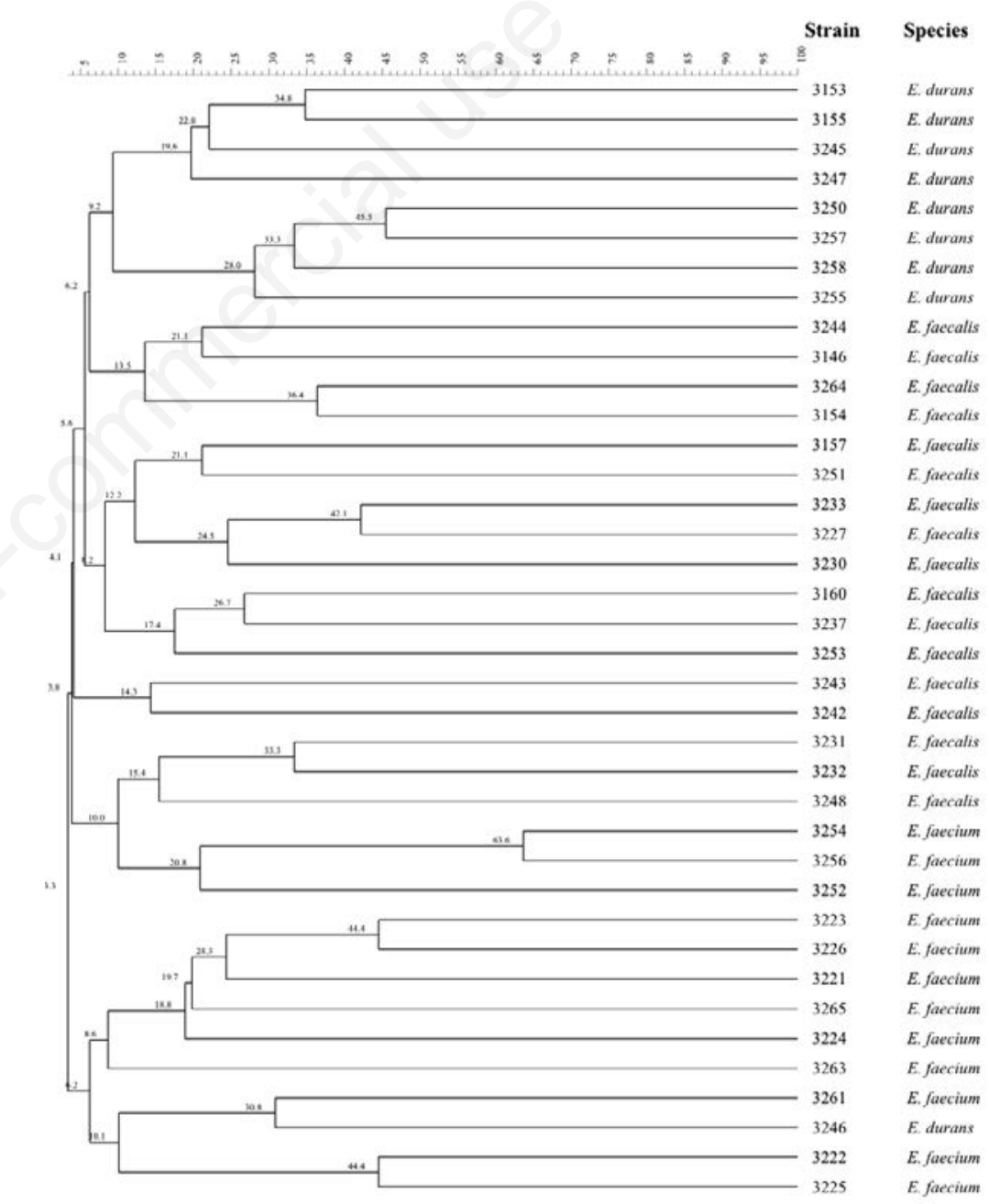

Figure 2. Dendrogram obtained with combined RAPD-PCR patterns of the LAB strains isolated from raw ewes' and cows' milk. Scale bar indicate the percentage of similarity. Abbreviations: E., Enterococcus. 
tified in this study was susceptible to streptomycin. These results confirmed an intrinsically resistance of enterococci to this antimicrobial agent as previously reported by Çitak, Yucel, and Orhan (2004). In particular, in this study, a considerable percentage of enterococci $(89.1 \%)$ isolated from Turkish white cheese were resistant to streptomycin at $10 \mu \mathrm{g}$. Moreover, the Enterococcus strains isolated in this study exhibited high percentages of resistance to quinupristin/dalfopristin $(23.7 \%)$ and tetracycline $(15.8 \%)$ and high percentages of intermediate susceptibility to ciprofloxacin $(50 \%)$ and erythromycin (53\%). These results confirmed as reported by Gaglio et al. (2016b) for the resistance to quinupristin/dalfopristin and by Silvetti, Morandi, and Brasca (2019) regarding intermediate susceptibility to erythromycin and ciprofloxacin of different strains of $E$. faecalis.

The high percentage of resistance registered for quinupristin/dalfopristin, was a surprising for the enterococci isolated from raw milk. Indeed, this antimicrobial agent is commonly used for the treatment of human infections (Allington and Rivey, 2001) and its use is not authorized in veterinary medicine. To this purpose, the presence of

Table 2. Antimicrobial resistance of the enterococcal isolates.

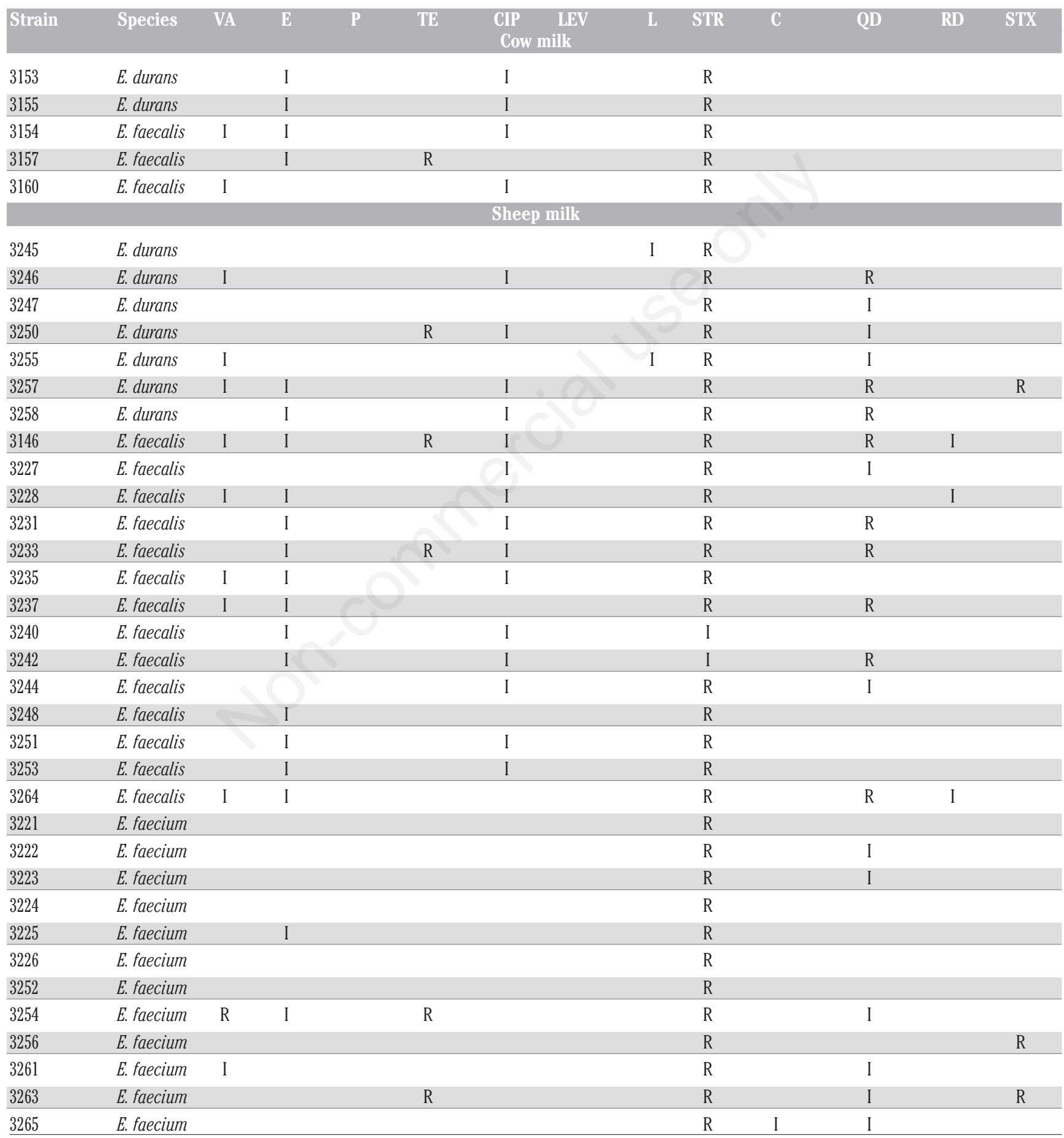

VA: vancomycin; E: erythromycin; P: penicillin; TE: tetracycline; ciprofloxacin: CIP: ciprofloxacin; LEV: levofloxacin; L: linezolid; STR: streptomycin; C: chloramphenicol; QD: quinupristin-dalfopristin; RD: rifampicin; STX sulphamethoxazole/trimethoprim. $\mathrm{R}=$ resistant; $\mathrm{I}=$ intermediate; no letter= susceptible. 
these strains in semi-extensive dairy sheep and cow farms could be due to cross-contaminations not associated with the animals. On the contrary, the resistance to tetracycline and sulphonamides is mainly due to the use of this antimicrobial agents in Sicilian farms veterinary practices. Regarding vancomycin, one strains was found resistant while eleven with intermediate susceptibility. The only stain resistant to vancomycin belonged to the specie $E$. faecium. Indeed, according to Russo et al. (2018), this resistance trait is common among E. faecium. As reported by Kang and co-workers (Kang, Kim, Chon, and Seo, 2017), our results exhibited high percentages (95\%) of Enterococcus strains susceptible to chloramphenicol. Regarding linezolid just two stains resulted resistant. These result is in accordance with what was reported by Hammad, Hassan, and Shimamoto (2004) who analyzed several enterococci isolated from Egyptian fresh raw milk cheese. In accordance to the published literature on antimicrobial resistance in enterococci isolated from dairy productions (Gaglio et al., 2016b; Silvetti et al., 2019), our results showed a higher percentage of Enterococcus strains susceptible to penicillins (penicillin) and fluoroquinolones (levofloxacin). Between these antimicrobial compounds, penicillins represents the most common therapeutic options for the treatment of enterococcal infections (Chow, 2000).

\section{Conclusions}

This study represents the first investigation on the antimicrobial resistance of enterococci isolated from raw ewes' and cows' milk samples collected from semi-extensive dairy sheep and cow farms of western Sicily.

The results of the present study confirmed that dairy enterococci might be a potential source for dissemination of antimicrobial resistances among bacteria in semi-extensive dairy sheep and cow farms of western Sicily.

This pointed out the relevance of informing dairy makers and veterinary regarding the antimicrobial use in order to mitigate problems of public health and veterinary medicine by reducing the potential impact of transmission of resistant bacteria to humans via the food chain.

However, further studies are being prepared to better characterize the safety of these enterococci in terms of detection of antimicrobial resistance genes, virulence as well as cellular toxicity.

\section{References}

Allington DR, and Rivey MP, 2001. Quinupristin/dalfopristin: a therapeutic review. Clin Ther 23:24-44.

Çitak S, Yucel N, and Orhan S, 2004. Antibiotic resistance and incidence of Enterococcus species in Turkish white cheese. Int J Dairy Technol 57:27-31.

Clinical and Laboratory Standards Institute (CLSI) 2017. Performance Standards for Antimicrobial Susceptibility Testing: 27th ed. CLSI Supplement M100. Wayne, PA: Clinical and Laboratory Standards Institute.

Chow JW, 2000. Aminoglycoside resistance in enterococci. Clin Infect Dis 31:58689.

Cruciata M, Gaglio R, Scatassa ML, Sala G, Cardamone C, Palmeri M, Moschetti G, La Mantia T, and Settanni L, 2018. Formation and characterization of early bacterial biofilms on different wood typologies applied in dairy production. Appl Environ Microbiol 84:e02107-17.

Cruciata M, Gaglio R, Todaro M, and Settanni L, 2019. Ecology of Vastedda della valle del Belìce cheeses: A review and recent findings to stabilize the traditional production. Food Rev Int 35:90103.

EFSA BIOHAZ Panel (EFSA Panel on Biological Hazards), 2016. Update of the list of QPS-recommended biological agents intentionally added to food or feed as notified to EFSA 4: Suitability of taxonomic units notified to EFSA until March 2016. EFSA J 14:4522.

Fernández L, Langa S, Martín V, Maldonado A, Jiménez E, Martín R, and Rodríguez JM, 2012. The human milk microbiota: origin and potential roles in health and disease. Pharmacol Res 69:1-10.

Foulquié Moreno M, Sarantinopoulos P, Tsakalidou E, and De Vuyst L, 2006. The role and application of enterococci in food and health. Int J Food Microbiol 106:1-24.

Franciosi E, Settanni L, Cologna N, Cavazza A, and Poznanski E, 2011. Microbial analysis of raw cows' milk used for cheese-making: influence of storage treatments on microbial composition and other technological traits. World J Microb Biot 27:171-80.

Franz CM, Holzapfel WH, and Stiles ME, 1999. Enterococci at the crossroads of food safety? Int J Food Microbiol 47:124.

Gaglio R, Francesca N, Maniaci G, Corona O, Alfonzo A, Giosuè C, Di Noto AM, Cardamone C, Sardina MT, Portolano B, and Alabiso M, 2016a. Valorization of indigenous dairy cattle breed through salami production. Meat Sci 114:58-68.

Gaglio R, Couto N, Marques C, Lopes MDFS, Moschetti G, Pomba C, and Settanni L, 2016b. Evaluation of antimicrobial resistance and virulence of enterococci from equipment surfaces, raw materials, and traditional cheeses. Int J Food Microbiol 236:10714.

Gaglio R, Francesca N, Di Gerlando R, Mahony J, De Martino S, Stucchi C, Moschetti G, and Settanni L, 2017. Enteric bacteria of food ice and their survival in alcoholic beverages and soft drinks. Food Microbiol 67:17-22.

Gaglio R, Cruciata M, Scatassa ML, Tolone M, Mancuso I, Cardamone C, Corona O, Todaro M, and Settanni L, 2019a. Influence of the early bacterial biofilms developed on vats made with seven wood types on PDO Vastedda della valle del Belìce cheese characteristics. Int J Food Microbiol 291:91-103

Gaglio R, Todaro M, Scatassa ML, Franciosi E, Corona O, Mancuso I, Di Gerlando R, Cardamone C, and Settanni L, 2019b. Transformation of raw ewes' milk applying "Grana" type pressed cheese technology: Development of extra-hard "Gran Ovino" cheese. Int J Food Microbiol 307:108277.

Giraffa G, 2002. Enterococci from foods. FEMS Rev. Microbiol 26:163-71.

Guarcello R, De Angelis, M, Settanni L, Formiglio S, Gaglio R, Minervini F, Moschetti G, and Gobbetti M, 2016a. Selection of amine-oxidizing dairy lactic acid bacteria and identification of the enzyme and gene involved in the decrease of biogenic amines. Appl Environ Microbiol 82:6870-80.

Guarcello R, Carpino S, Gaglio R, Pino A, Rapisarda T, Caggia C, Marino G, Randazzo CL, Settanni L, and Todaro M, 2016b. A large factory-scale application of selected autochthonous lactic acid bacteria for PDO Pecorino Siciliano cheese production. Food Microbiol 59:66-75.

Guzman Prieto AM, van Schaik W, Rogers MR, Coque TM, Baquero F, Corander J, and Willems RJ, 2016. Global emergence and dissemination of enterococci as nosocomial pathogens: attack of the clones? Front Microbiol 7:788.

Hammad AM, Hassan HA, and Shimamoto T, 2015. Prevalence, antibiotic resistance and virulence of Enterococcus spp. in Egyptian fresh raw milk cheese. Food Control 50:815-20.

Jackson CR, Fedorka-Cray PJ, and Barrett JB, 2004. Use of a genus- and speciesspecific multiplex PCR for identifica- 
tion of enterococci. J Clin Microbiol 42:3558-65.

Kang II-B, Kim DH, Chon JW, and Seo $\mathrm{KH}, 2017$. Effect of microbial control measures on farmstead cheesmaking and antimicrobial resistance of Staphylococcus aureus and Enterococcus spp. isolates. J Food Saf 38:e12432.

Licitra G, Ogier JC, Parayre S, Pediliggieri C, Carnemolla TM, Falentin H, Maden MN, Carpino S, and Lortal S, 2007. Variability of bacterial biofilms of the "tina" wood vats used in the Ragusano cheese-making process. Appl Environ Microbiol 73:6980-87.

Macaluso G, Fiorenza G, Gaglio R, Mancuso I, and Scatassa ML, 2016. In vitro evaluation of bacteriocin-like inhibitory substances produced by lactic acid bacteria isolated during traditional Sicilian cheese making. Ital J Food Saf 5:5503.

Mannu L, Paba A, Daga E, Comunian R, Zanetti S, Dupré I, and Sechi LA, 2003. Comparison of the incidence of virulence determinants and antibiotic resistance between Enterococcus faecium strains of dairy, animal and clinical origin. Int J Food Microbiol 88:291-304.

Russo N, Caggia C, Pino A, Coque TM, Arioli S, and Randazzo CL, 2018. Enterococcus spp. In Ragusano PDO and Pecorino Siciliano cheese types: a snapshot of their antibiotic resistance distribution. Food Chem Toxicol 120: 277-86.

Scatassa ML, Gaglio R, Cardamone C,
Macaluso G, Arcuri L, Todaro M, and Mancuso I, 2017. Anti-Listeria activity of lactic acid bacteria in two traditional Sicilian cheeses. Ital J Food Saf 6:1317.

Silvetti T, Morandi S, and Brasca M, 2019. Does Enterococcus faecalis from traditional raw milk cheeses serve as reservoir of antibiotic resistance and pathogenic traits? Foodborne Pathog Dis 16:359-67.

Sitzia M, Bonanno A, Todaro M, Cannas A, Atzori AS, Francesconi AHD, and Trabalza-Marinucci M, 2015. Feeding and management techniques to favour summer sheep milk and cheese production in the Mediterranean environment. Small Rumin Res 126:43-58. 